\title{
Evaluation of the Role of High Resolution Computed Tomography and Magnetic Resonance Cisternography in Preoperative Identification of Skull Base Defect in Cases of Cerebrospinal Fluid Rhinorrhea
}

\author{
Mohamed $E^{a}$, Ibrahim $A A^{b}$, Ihab EA ${ }^{c}$, Elwany $S^{b}$, Hassab $M H^{b}$, Khamis $H M^{a}$ \\ ${ }^{a}$ Department of Diagnostic Imaging, Alexandria University, Egypt. \\ ${ }^{b}$ Department of Otorhinolaryngology-Head \& Neck Surgery, Faculty of Medicine, Alexandria University, Egypt. \\ 'Department of Otorhinolaryngology-Head \& Neck Surgery, Faculty of Medicine, AIMST University, \\ Malaysia.
}

\begin{abstract}
Introduction: The New Zealand White (NZW) rabbit model is commonly used for the research of posterolateral intertransverse lumbar arthrodesis. The standard approach reported by many researchers are a muscle splitting approach through the intermuscular plane between multifidus and longissimus muscles. Methods: In this research the fusion bed was exposed by retracting the three groups of paraspinal muscles (multifidus, longissimus and iliocostalis) medially. Results: This alternative method showed good exposure of the transverse processes and intertransverse membrane with less bleeding from the muscles observed. No death due to surgical complication was observed out of twenty-four rabbits operated. Conclusion: An easy and safe method for performing posterolateral intertransverse lumbar arthrodesis was demonstrated and recommended as an alternative approach for surgery on New Zealand White rabbits.
\end{abstract}

KEYWORDS: Lumbar vertebrae, arthrodesis, surgical approach, spinal fusion, NZW rabbits.

\section{INTRODUCTION}

Cerebrospinal fluid (CSF) leak or fistula describes the egress of CSF from the intracranial cavity through an osseous defect within the skull base. It implies a breach or disruption of the underlying dura mater and adherent pia-arachnoid mater, resulting in a communication between the intracranial cavity and either the nasal or middle ear cavity. The condition was first described as a pathologic entity in 1899 by Clair Thompson. Over the subsequent decades, the clinical importance of a CSF leak was recognized, demonstrating affected patients to be at risk of developing intra-cranial complications. ${ }^{1}$

CSF leaks have been classified according to the aetiology and presentation. The most widely accepted classification system, described by Ommaya in 1960, fell under two categories: traumatic and non-traumatic leakage. ${ }^{2}$ A third category, spontaneous leaks, describes CFS leakage in patients with no history of trauma or other predisposing etiology. ${ }^{3-5}$ Post-traumatic CSF leaks account for nearly $90 \%$ of all cases, with approximately $80 \%$

Corresponding author:

Ahmad A. Ibrahim, MD.

19 Sorya Street, Roushdi,

Alexandria, Egypt

Phone: +203 5454074

E-mail: aly284@hotmail.com manifesting as rhinorrhea and $20 \%$ as otorrhea. CSF leaks secondary to skull base fracture complicate about $1 \%-3 \%$ of all closed head injuries. Also included in this category are the iatrogenic CSF leaks, which were caused frequently by the otolaryngologic or neurosurgical procedures. The most common sites of traumaticinjury involve the anterior cranial fossa, with fractures through the frontal sinus or cribriform plates of the ethmoid bones. Central skull base fractures occur at the sphenoid sinus or sella floor. Lateral skull base fractures occur in the temporal bone, with communication of CSF into the mastoid complex and middle ear cavity. These fractures may cause middle ear CSF effusion and drainage into the nasopharynx or result in frank CSF otorrhea if there is a tympanic membrane perforation. ${ }^{4-7}$

Non-traumatic CSF leaks can occur in both normal and elevated intracranial pressure states. This include cases with skull-base tumours, infections, or congenital lesions. ${ }^{8-10}$

Finally, spontaneous, or primary, CSF fistula is considered a separate entity describing patients with no other discernible aetiology for their CSF leak. Demographically, these leaks most frequently occur in obese middle-aged women and co-exist with a small encephalocele. ${ }^{11-14}$ Spontaneous CSF fistula can occur anywhere in the skull base, but is most common at the ethmoid roof, cribriform plate, or at two locations in the sphenoid sinus, perisellar or at the inferolateral or pterygoid recesses. ${ }^{11,14-17}$ Successful surgical repair of CSF leaks depends on accurate pre- 
operative localization of the site of the defect. Several diagnostic techniques advocated for such localization can be difficult to perform. High resolution computed tomography (HRCT) provides thin overlapping cuts in both the axial and coronal planes and enables good definition of bony structures.

On plain CT scans, a CSF leak may appear as an opacification of a sinus, but this may be CSF, a mucosal reaction, a meningocele, or percolated CSF from a distal breach. ${ }^{18-22}$ For many years, CT cisternography has been considered to be the gold standard for detecting CSF leaks. Its detection rate ranges between 40 and $92 \%$, but, the leak must be active. This modality is therefore unsuitable for patients with intermittent leaks. Furthermore, it is contraindicated in patients with high intracranial pressure and in those with spinal disorders. Its acceptability is low and accurate results are highly operator dependent. ${ }^{20-24}$

Magnetic resonance imaging (MRI) cisternography depends on heavily $\mathrm{T2}$-weighted sequences with fat suppression. CSF appears as a bright signal without the need to inject contrast media intrathecally. Additionally, MRI details the intracranial anatomy and pathology in multiple planes within a relatively short time. The main disadvantage of MRI is its poor spatial resolution and lack of bony details. ${ }^{22-24}$ Thus, CT and MRI seem to be complementary in the diagnosis of CSF leaks. ${ }^{17,19,21-22}$

This study was conducted to determine the reliability and accuracy of combining HRCT and MR Cysternography to identify the sites of CSF leakage without the need for $\mathrm{CT}$ cisternography.

\section{MATERIALS AND METHODS}

Thirty one patients were included in this study after approval of the ethical review board of Alexandria University. The patients were proven to have CSF rhinorrhea based upon the laboratory and radiological findings. All suspected fluid was collected and tested for B2-transferrin and trace protein for verification of CSF leak, which was positive in all cases. The study included combined evaluation by HRCT and 3D T2 weighted MR Cisternography to identify the sites of the leakage in all patients.

\section{High resolution CT (HRCT)}

Non contrast HRCT involves rapid, continuous volumetric acquisition of raw data by using Toshiba Aquilion machine. The settings used were 16 slices thin collimation $(16 \times 1 \mathrm{~mm})$, allowing for isotropic voxels, which leads to improved resolution for three-dimensional and multiplanar reformations, tube rotation time $400 \mathrm{msec}$, tube voltage of $120 \mathrm{Kv}$ and current of $300 \mathrm{mAs}$. Thus, with multidetector CT, images can be acquired in only the axial plane, and then the raw data can be reformatted into additional planes without a compromise in image resolution.

\section{MRI examination}

MR Cisternography typically involves heavily T2-weighted fast spin echo sequences with fat suppression and subtraction of the adjacent background tissue signal to enhance conspicuity of the fistulous tract, or CSF column. The fast spin echo sequences have decreased susceptibility artefacts at the air-bone interface of the skull base, compared with conventional T2-weighted imaging. Overlapping heavily T2-weighted fast spin-echo sequences with fat suppression are then performed at TR $=1200$ msecs, $\mathrm{TE}=263$ msecs, slice $=0.6 \mathrm{~mm}$ and $\mathrm{FOV}=$ $20 \mathrm{~cm}$. The proponents of MR Cisternography state that the non-invasive and non-ionizing technique can localize the actual site of a fistulous tract, which may be particularly helpful in patients who have multiple potential fracture sites or osseous defects at CT.

Statistical analysis was carried out using the SPSS software, version 11 (Chicago, Illinois).

\section{RESULTS}

The study included 31 patients. Twenty four patients were females. Their ages range from 18 to 70 years. Twenty-seven patients had a single defect, the most common site was at the ethmoidal roof (19 patients), followed by the sphenoid (6 patients) and frontal area (2 patients). Four patients had multiple defects; three in the ethmoid/sphenoid area and one patient with frontal/ethmoid/sphenoid area. Two patients out of the 31 with bony defects had meningo-encephalocele (Figure 1).

Most of the patients ( 20 cases) had idiopathic CSF leak with a skull base defect (Figures 2, 3A and B). Six patients had a post-traumatic or post-operative etiology. Four patients were confirmed clinically to have idiopathic intracranial hypertension (IIH) with bilateral optic hydrops (Figure $3 \mathrm{~A}$ ). One patient was diagnosed as schwannoma of the trigeminal nerve, which erodes the posterolateral wall of the sphenoid sinus (Figure $3 \mathrm{~B}$ ).

In the 31 patients, the site of the leak could be easily detected by combined CT and MR Cisternography. The combined HRCT and 3D T2 weighted MR Cisternography images had a sensitivity of $96 \%$ and specificity of $90 \%$ in detection of the skull base defects. The positive predictive value was $90 \%$, and the negative predictive value was $96 \%$. 

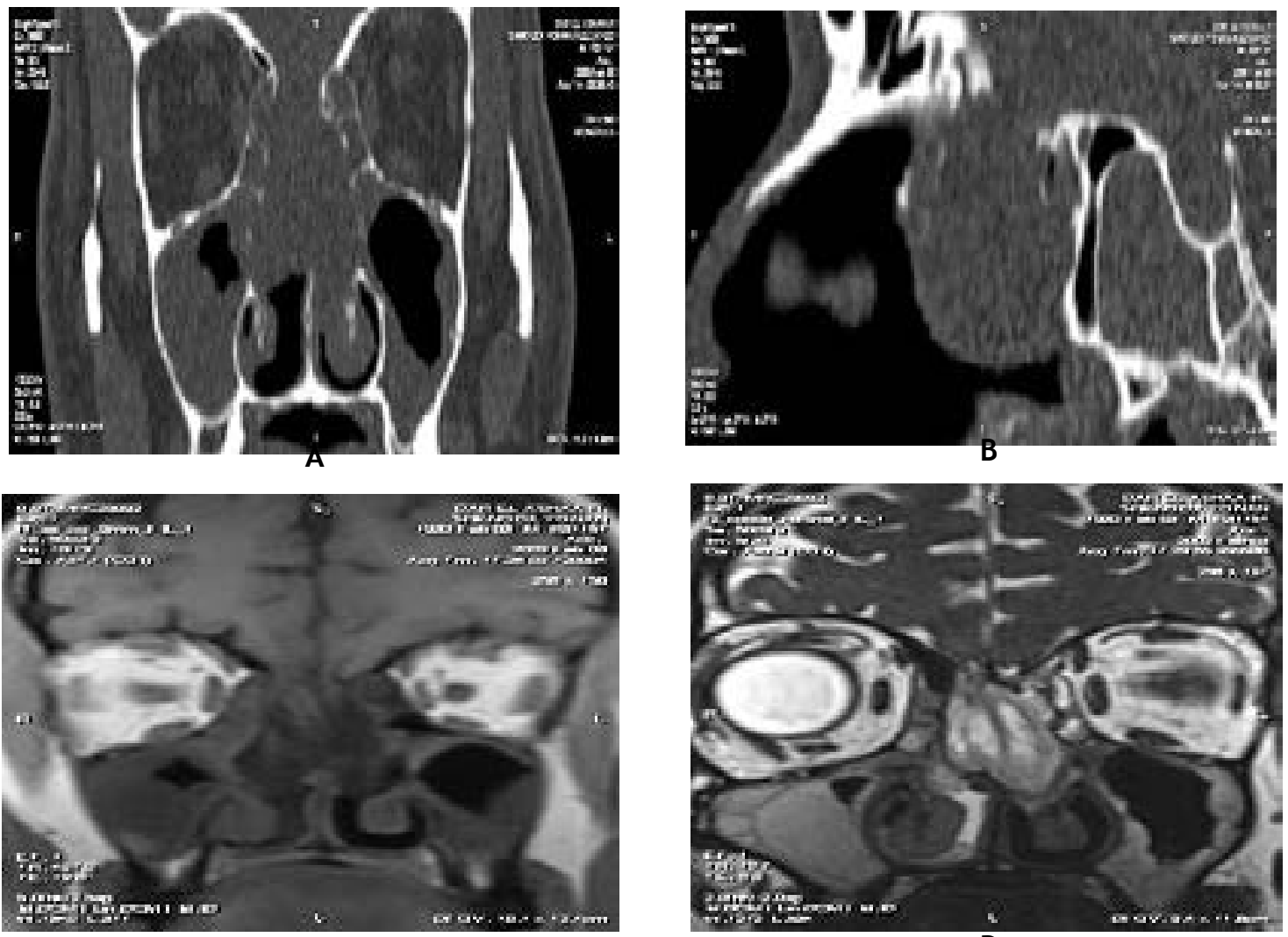

C

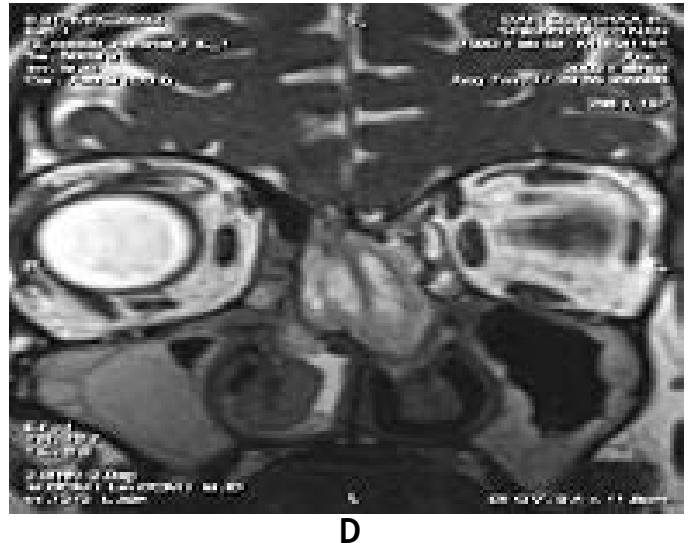

Figure 1. 18-year-old male, presented with right sided CSF rhinorrhea. (A and B) MDCT revealed a large ethmoidal roof defects with polypoid opacity filling the ethmoidal and nasal cavity. MRI (C\&D) was performed to verify the nature of the opacity and revealed large meningoencephalocele with gliotic gyrus rectus.

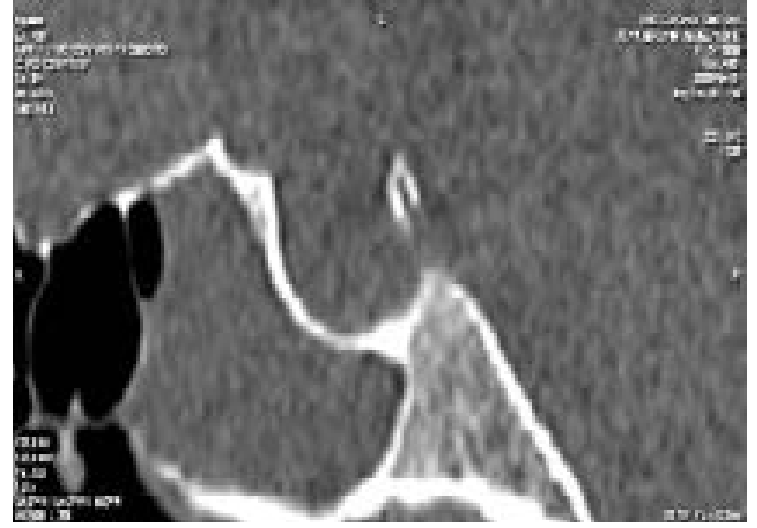

A

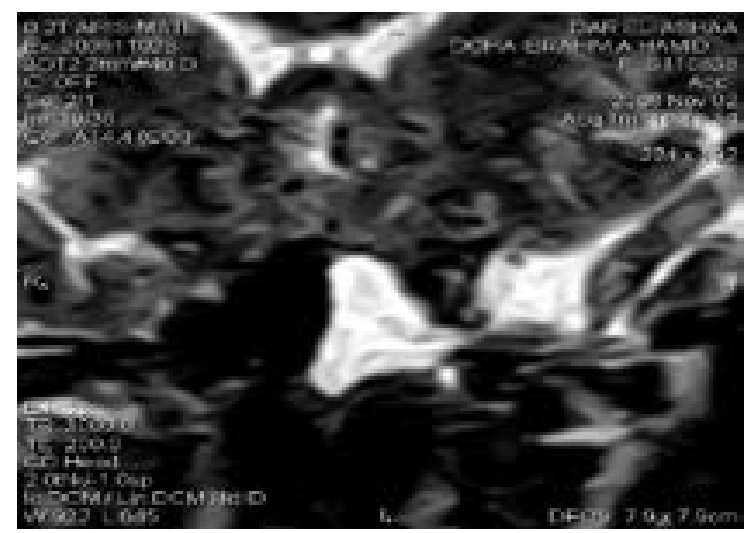

C

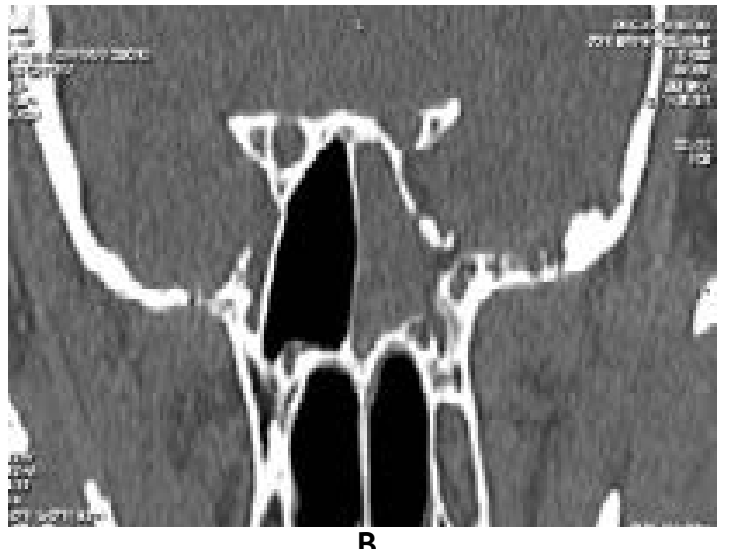

B

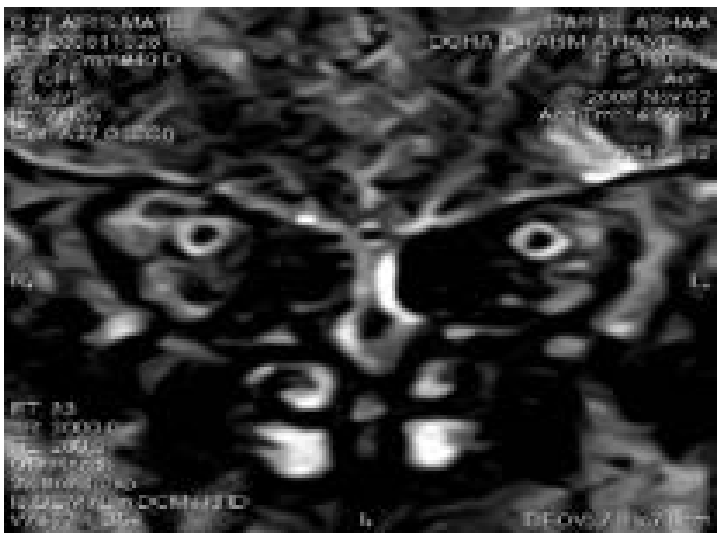

D

Figure 2. 42 years-old female presented with left sided rhinorrhea. (A\&B) HRCT revealed two skull base defects locatedat thelateral recess of theleftsphenoidsinusanddorsumsellae. (C)3-DT-2 weightedMRCisternographyrevealed filling of the left sphenoid sinus by CSF intensity fluid continuous with the subarachnoid space through the described defect. (D) Optic hydrops also noted that indicate idiopathic intracranial hypertension. 

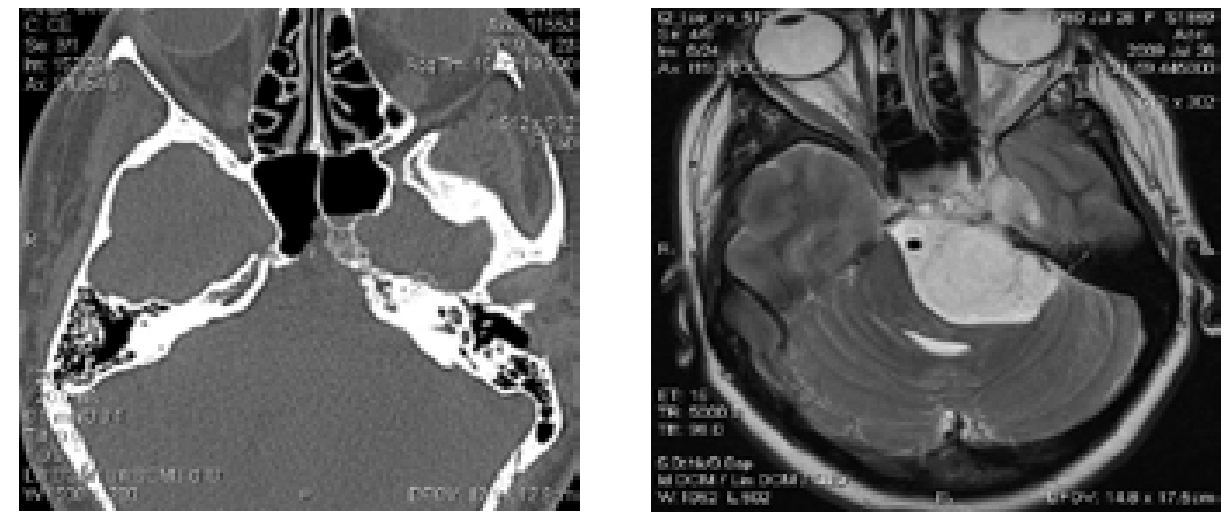

Figure 3. (Left) Axial high resolution CT shows a bony defect in the posterior wall of the right sphenoid sinus. (Right) Axial T-2 weighted MRI shows a large space occupying hyperintense lesion occupying the left cerebellopontine angle and cavernous sinus (Trigeminal Schwannoma).

\section{DISCUSSION}

CSF rhinorrhea is a communication between the subarachnoid space and the nasal cavity. ${ }^{25}$ Accurate localization of the site of the CSF fistula is challenging for the neuroradiologists as well as for the surgeons. Precise identification of a CSF fistula helps in surgical planning and enhances the chances of a successful dural repair. Several laboratory and radiological methods have been used for the preo-perative identification and localization of the defect. These methods have been associated with various rates of success in locating CSF fistulas. ${ }^{16,18,22}$

The main laboratory investigation is the identification of the B2 transferrin in the liquid sample collected from the nose. It is a rapid test and requires just a few millilitres of liquid, and has a sensitivity of $97.7 \%{ }^{26}$ The main limitation of this test is that rhinorrhea must be active and even in cases of intermittent rhinorrhea, it is not always easy to collect the fluid sample. ${ }^{26-27}$

Nowadays, HRCT is the best imaging study for localizing skull base defects and CSF leaks, as it is possible to obtain very thin slices $(0.5 \mathrm{~mm})$ with a bone algorithm. The examination takes a short time. It allows for a complete study in the axial, coronal, and sagittal planes in supine position with reconstruction. In some cases, the coronal section has to be perpendicular to the hard palate to show the entire temporal bone and exclude the defect in the tegmen tympani or the roof of the mastoid when CSF flow from the middle ear or the mastoid through the eustachian tube that could simulate rhinorrhea. ${ }^{28}$ Furthermore, CT data can provide intra-operative image guidance for the endoscopic surgical repair. The findings suggestive of a CSF leak include a skull base bone defect and an air-fluid level or opacification of the contiguous sinus. CT is helpful in evaluation of non-traumatic causes of CSF leak, such as an intracranial or skull base tumour, or meningocele and/or meningo-encephalocele, which may then require further evaluation with MR imaging.

Occasionally, CT may be inadequate because of a small defects can be missed through the partial volume effect, leading to both false-negative and false-positive findings. Inaccuracies can be minimized by using the thinnest sections possible but at the expense of a significantly higher radiation dose to the eye. ${ }^{29-30}$

Detection of a small skull base defect on CT is not necessarily related to a connection between the nasal cavity and the intracranial compartment. In HRCT, the diagnostic criteria are not specific if pre-existing paranasal sinus inflammation has been reported. Similarly, multiple osseous defects and adjacent opacified sinuses, especially in the posttraumatic patient may lead to false-positive diagnoses. In this instance, further imaging and evaluation may be necessary to localize the site of the leak with CT cisternography. ${ }^{14}$

CT cisternography examination is reserved for patients with multiple skull base fractures and/or defects, patients with negative CT scans, or those in whom the diagnosis is questionable. This technique traditionally involves obtaining thin-section corona and axial CT images in prone position through the region of interest (maxillofacial and temporal region), both before and after intrathecal contrast material injection.

Approximately 3-10 $\mathrm{mL}$ of an iodinated nonionic low-osmolar contrast agent is administered by means of lumbar puncture, and the patient is placed in a Trendelenburg position to opacify the basal cisterns, followed by CT imaging. Manoeuvres that provoke an active leak, such as sneezing or head hanging, are performed prior to the CT portion of cisternography. Post-contrast images are then compared with the pre-contrast images.

A positive result involves the presence of a skull base defect and contrast opacification within the sinus or nasal cavity. If the sinus contents do not visually show increase attenuation after cisternography, objective attenuation measurements in Hounsefield Units (HU) by drawing a region of interest may be used. An increase in Hounsfield units of 50\% or greater after CT cisternography is considered a 
positive study for a CSF leak. ${ }^{4}$

CT cisternography is only positive during the active leak. It is considered to be the standard of reference for the diagnosis of CSF fistula. However, it is an invasive procedure with low acceptance from patients, expensive, with inherent risk of infection and lumbar CSF leaks. The specificity and sensitivity of CT cisternography vary between 40 and $91 \% .{ }^{21}$ Contrast agent used here are iohexol and iopamidol which has low-osmolarity, and associated with lower incidence of the major side effects (headache, meningeal irritation and seizures), compared to metrizamide which is routinely used before. In many institutions, unenhanced CT is performed before CT cisternography. However, this procedure also double the radiation exposure and some patients may require delayed images, which will further increases the radiation exposure. ${ }^{21-22}$

Another invasive method is the intrathecal fluorescein which is most commonly used as an adjunct to intraoperative localization of a skull based defect. The process involves standard lumbar puncture followed by withdrawal of $10 \mathrm{cc}$ of CSF, which is mixed with $0.2-0.25$ cc of $5 \%$ fluorescein. After 30 minutes, a yellow fluid leaking into the nose is observed endoscopically. ${ }^{31}$

MRI techniques offer non-invasive methods of imaging a CSF leak and are indicated to assess possible encephalocele or meningo-encephalocele through a bone defect that may be difficult to differentiate from obstructed secretions on CT. MRI with high resolution, 3-mm, axial, coronal, and sagittal T1 and T2-weighted sequences is mandatory to formulate a differential diagnosis in cases with active CSF leak. It is always performed if there are an osseous defect and complete opacification of an adjacent sinus in the patient with a possible CSF leak, which is more sensitive than CT in detecting continuity between the nasal and cranial cavities. Demonstration of high signal intensity fluid extending from the subarachnoid space directly into adjacent paranasal sinuses or herniation of brain into a sinus through a bone defect is the principal diagnostic criteria. When there is a lesion with a very narrow neck, the examination is valuable in differentiating herniated brain from inflammatory nasal masses, nasal gliomas, or nasal dermal sinus cysts and to identify the contents of the herniated mass. ${ }^{32} \mathrm{MRI}$ without contrast medium has been used by many authors for investigation of CSF fistulae. Shetty et al. ${ }^{21}$ claimed an accuracy of $89 \%$, but Hegarty et al. ${ }^{33}$ reported a relatively high (40\%) false-positive rate. The presence of inflammatory soft tissue in the paranasal sinuses increases the rate of false-positive studies; with false negatives are also reported. ${ }^{22}$

MR cisternography is a non-invasive technique demonstrating CSF-like signal in multiple planes without the disadvantage of ionizing radiation. Its accuracy in patients with active CSF rhinorrhea is $86 \%$. The primary disadvantages of MRI are its poor spatial resolution compared with HRCTs and the absence of bony details. ${ }^{22-32}$

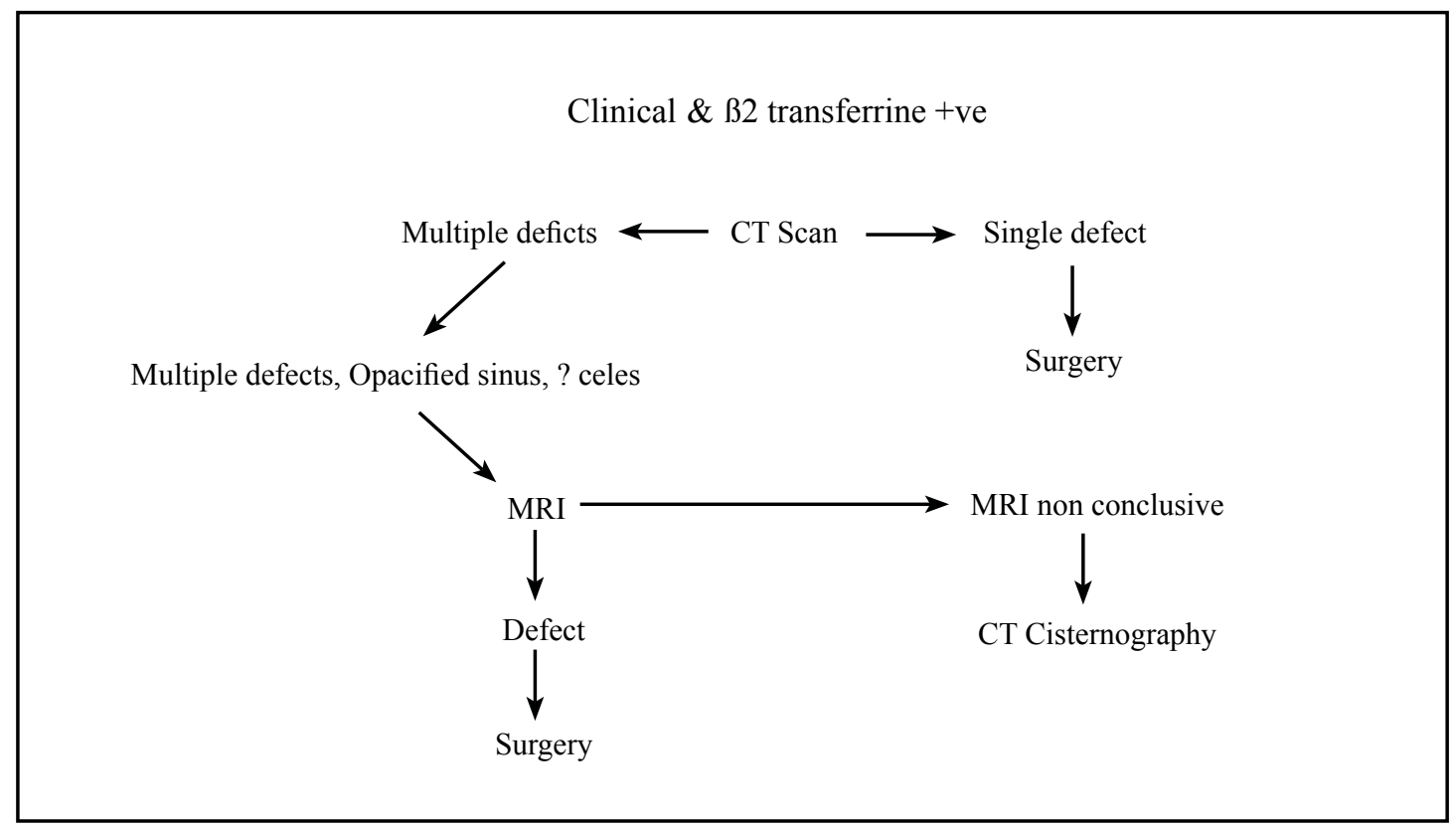

The suggested radiological algorithm for cases presented with CSF rhinorrhea 
In this study, we combined both techniques of HRCT scans and 3D T2-weighted MRI techniques. Both are standardized, non-invasive, and readily available. Also, we believe that fat suppression increases the complicity of contrast medium leakage due to the saturation of medullary bone fat at the skull base and nasal cavity, which might conceivably be confused with leakage.

In our study, the combined HRCT and 3D T2-weighted MRI detected bone defect in $96 \%$ of the cases. CT cisternography is only needed in equivocal cases. From our results, we recommend the following algorithm (figure) for localization of the site of the defect in cases of CFS leak:

-When the B2-transferrin test is positive and multidetector $\mathrm{CT}$ scan shows a single osseous defect, no other imaging is necessary to direct the repair. This is the most common scenario.

-If the B2-transferrin test is positive and the HRCT scan shows more than one osseous defect, an opacified sinus, or suspected cephaloceles or meningoceles; MRI will be obtained to determine the site of leakage.

- If MRI \& MDCT are non-conclusive, further CT cisternography is performed.

\section{REFERENCES}

1. Aarabi B, Leibrock LG. Neurosurgical approaches to cerebrospinal fluid rhinorrhea. Ear Nose Throat J 1992;71: 300-5.

2. Wax MK, Ramadan $\mathrm{HH}$, Ortiz O, Wetmore SJ. Contemporary management of cerebrospinal fluid rhinorrhea. Otolaryngol Head Neck Surg 1997;116:442-9.

3. Yerkes SA, Thompson DH, Fisher WS. Spontaneous cerebrospinal fluid rhinorrhea. Ear Nose Throat J 1992;71:318-20.

4. Zlab MK, Moore GF, Daly DT, Yonkers AJ. Cerebrospinal fluid rhinorrhea: a review of the literature. Ear Nose Throat J 1992;71:314-7.

5. Schlosser RJ, Bolger WE. Nasal cerebrospinal fluid leaks. J Otolaryngol 2002;31:28-37.

6. Yilmazlar S, Arslan E, Kocaeli H, et al. Cerebrospinal fuid leakage complicating skull base fractures: analysis of 81 cases. Neurosurg Rev 2006;29:64-71.

7. Schlosser RJ, Bolger WE. Nasal cerebrospinal fluid leaks: critical review and surgical considerations. Laryngoscope 2004;114:255-65.

8. Chang DW, Langstein HN, Gupta A, et al. Reconstructive management of cranial base defects after tumor ablation. Plast Reconstr Surg 2001;107:1346-55; discussion1356-7.

9. Lim BY, Pang KP, Low WK, Tan HM. CSF Volume 11 Number 2, Dec 2012 otorrhea complicating temporal bone osteoadionecrosis in a patient with nasopharyngeal carcinoma. Ear Nose Throat J 2005; 84(1):39-40.

10. Holodny Al, Arutiunov NV, Kornienko VN, et al. Aqueductal stenosis leading to herniation of the frontal horn of the lateral ventricle into the frontal sinus. J Comput Assist Tomogr 1997; 21:837-9.

11. Lai SY, Kennedy DW, Bolger WE. Sphenoid encephaloceles: disease management and identification of lesions within the lateral recess of the sphenoid sinus. Laryngoscope 2002; 112:1800-5.

12. Lindstrom DR, Toohill RJ, Loehrl TA, Smith TL. Management of cerebrospinal fluid rhinorrhea: the Medical College of Wisconsin experience. Laryngoscope 2004;114:969-74.

13. Clark D, Bullock P, Hui T, Firth J. Benign intracranial hypertension: a cause of CSF rhinorrhea. J Neurol Neurosurg Psychiatry 1994;57:847-9.

14. Schlosser RJ, Woodworth BA, Wilensky EM, Grady MS, Bolger WE. Spontaneous cerebrospinal fluid leaks: a variant of benign intracranial hypertension. Ann Otol Rhinol Laryngol 2006;115:495-500.

15. Gacek RR. Arachnoid granulation cerebro spinal fluid otorrhea. Ann Otol Rhinol Laryngol 1990; 99:854-62.

16. Shetty PG, Shroff MM, Fatterpekar GM, Sahani DV, Kirtane MV. A retrospective analysis of spontaneous sphenoid sinus fistula: MR and CT findings. AJNR Am J Neuroradiol 2000;21:337-42.

17. Schmerber S, Boubagra K, Cuisnier O, Righini C, Reyt E. Methods of identifcation and localization of ethmoid and sphenoid osteomeningeal breaches. Rev Laryngol Otol Rhinol 2001;122:13-9.

18. Eljamel MS, Pidgeon CN. Localization of inactive cerebrospinal fistulas. J Neurosurg 1995;83:795-8.

19. Sillers MJ, Morgan CE, El Gammal T. Magnetic resonance cisternography and thin coronal computerized tomography in the evaluation of cerebrospinal fluid rhinorrhea. Am J Rhinol 1997;11:387-92.

20. Simmen D, Bischoff T, Schuknecht B. Experiences with assessment of frontobasal defects, a diagnostic concept. Laryngorhinootologie 1997;76:583-7.

21. Shetty PG, Shroff MM, Sahani DV, Kirtane MV. Evaluation of high-resolution CT and MR cisternography in the diagnosis of cerebrospinal fluid fistula. Am J Neuroradiol 1998;19:633-9.

22. Lund VJ, Savy L, Lloyd G, Howard D. Optimum imaging and diagnosis of cerebrospinal fluid rhinorrhea. J Laryngol Otol 2000;114:988-92.

23. Eljamel MS, Pidgeon CN, Toland J, et al. 
MRI cisternography, and the localization of CSF fistula. Br J Neurosurg 1994;8:433-7.

24. Murata Y, Yamada I, Suzuki S. MRI in spontaneous cerebrospinal fluid rhinorrhea. Neuroradiology 1995;37:453-5.

25. Ramsden JD, Corbridge R, Bates G.

Bilateral cerebrospinal fluid rhinorrhea.

J Laryngol Otol 2000;114:137-8.

26. Skedros DG, Cass SP, Hirsch BE, Kelly RH. Beta-2transferrin assay in clinical management of cerebrospinal fluid and perilymphatic fluid leaks. J Otolaryngol 1993;22:341-44.

27. Ryall RG, Peacock MK, Simpson DA. Usefulness of beta-2-transferrin assay in the detection of cerebrospinal fluid leaks following head injury. J Neurosurg 1992;77:737-39.

28. Castelnuovo P, Mauri S, Locatelli D. Endoscopic repair of cerebrospinal fluid rhinorrhea: learning from our failure. Am J Rhinol 2001;15:333-42.

29. Locatelli D, Castelnuovo P, Santi L, et al. Endoscopic approaches to the cranial base: prospective and realities. Childs Nerv Syst 2000;16:686-91.

30. Bibas AG, Skia B, Hickey SA. Transnasal endoscopic repair of cerebrospinal fluid rhinorrhea. Br J Neurosurg 2000;14:49-52.

31. Eljamel MS, Pidgeon CN, Toland JB, Phillips J, O_Dwyer AJ. MRI cisternography and the localization of CSF fistulae. Br J Neurosurg 1194;8:433-7.

32. Eljamel MS, Pidgeon CN. Localization of inactive cerebrospinal fluid fistula. J Neurosurg 1995;83:795-8.

33. Hegarty SE, Millar JS. MRI in the localization of CSF fistulae: is it of any value? Clin Radiol 1997;52:768-70. 